\title{
TESTING A BIOLOGICAL WEAPON DETECTION SYSTEM IN A DISTRIBUTED VIRTUAL REALITY ENVIRONMENT
}

\author{
Dr. Paul Beckman \\ San Francisco State University \\ San Francisco, California, USA
}

\begin{abstract}
A study called Bio-Sim was sponsored by the U.S. Army to examine the LR-BSDS (Long-Range Biological Standoff Detection System), a laser-based biological cloud detector. Testing of the device was performed in a distributed virtual reality environment (DVRE). The three primary objectives of the study were to: 1) determine appropriate tactics, techniques, and procedures for use of the LR-BSDS, 2) gain a better understanding of those battlefield situations and characteristics that limit the effective use of the LR-BSDS, and 3) demonstrate the potential to use a DVRE simulation for training using the LR-BSDS. This paper will focus on the third of those objectives.

The study consisted of three phases. The first phase involved calculating theoretical concentration limits of a dispersed biohazard as a function of time, dispersion concentration, and distance to sensor. The second phase resulted in a set of abbreviated bio-cloud detection missions run in a DVRE. The third phase was a set of full-length human-in-the-loop trial missions run by trained LR-BSDS operators and helicopter flight crews, using a DVRE and computer-based simulators for the LR-BSDS, helicopter, biocloud dispersion, and bio-cloud transport.
\end{abstract}

\section{Background of the BIO-SIM Study}

The United States Army's Chemical and Biological Defense Command, now called the Soldier and Biological Chemical Command, initiated the Bio-Sim study. The purpose of the study was to learn more about appropriates uses of the LR-BSDS [Condatore et al., 1999] a laser-based helicopter-borne device that detects airborne biological hazard clouds. The LR-BSDS had been in use by the U.S. Army, but there had been no definitive testing of it in real or realistic battle situations. The culmination of the BioSim study was a set of 35 complete bio-detection missions flown by trained LR-BSDS operators and helicopter flight crew, using a distributed virtual reality environment.

\subsection{LR-BSDS}

The LR-BSDS uses LIDAR (light detection and ranging) technology [Mendonsa, 1997], pulsing a laser out the side of a helicopter (UH-60). The laser beam is aimed somewhat below horizontal, and is moved up and down in the LR-BSDS housing. This causes the beam to trace a sinusoidal path on the earth's surface at some distance out from the helicopter. The LR-BSDS has controls that allow the operator to change the near and far extent of the sinusoidal beam traced on the ground, to reduce the error caused by helicopter roll, and to input changes to the altitude of the helicopter. When the laser beam impacts a particle of a particular size (related to the frequency of the laser beam), that particle causes 
some backscatter of the laser beam energy to be directed back at the helicopter. A 24-inch receiving device similar to a telescope is mounted in the helicopter, directly next to the laser. This receiver picks up the backscatter, amplifies it, and integrates the signal over time to give the LR-BSDS operator a "sliced" view of the space cut by the laser beam. A trained operator can then look at that "sliced" view on LRBSDS screen and be able to distinguish a biological hazard cloud. A bio-cloud can be recognized based on characteristics such as the clouds extent in the $\mathrm{x}, \mathrm{y}$, and $\mathrm{z}$ direction, and on the cloud edges and internal concentration changes. The LR-BSDS screen (see Figure 1) is divided into two panes, the upper of which shows the instantaneous signal impinging on the receiver, and the lower of which shows the most recent slice of space cut by the laser. The LR-BSDS operator can highlight a small rectangular section of the lower screen. That rectangular section is automatically updated and displayed on the right side of the LRBSDS screen. The LR-BSDS operator can then view a time-series of cross-sections of an area of particular interested.

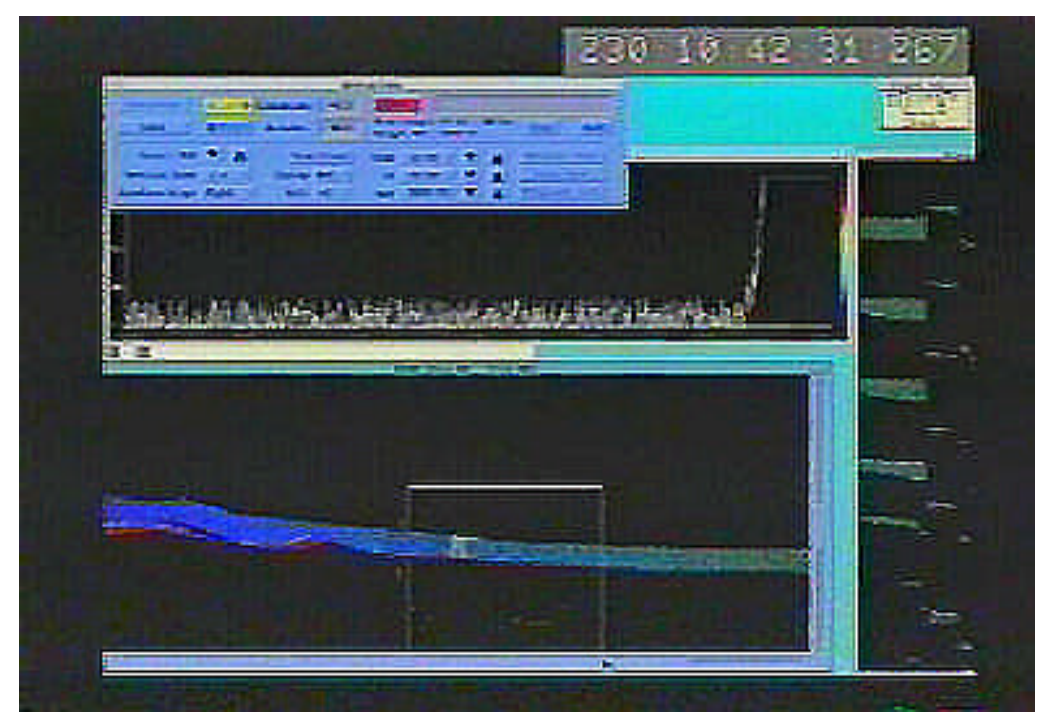

Figure 1: LR-BSDS screen capture (from VHS videotape) ${ }^{1}$

\subsection{STUDY OBJECTIVES}

One of the three Bio-Sim study objectives was to find those methods of using the LR-BSDS so that the bio-cloud mission would have the greatest chance for success while maximizing the survivability of the aircraft and crew. This objective arose from the imperative to improve FM 3-101-6, ("Tactics, Techniques, and Procedures for the Long-Range Biological Standoff Detection System [LR-BSDS]"), describing use of the LR-BSDS. FM 3-101-6 is used by the U.S. Army to explain to soldiers how to best use the LR-BSDS in support of detecting and identifying suspect biological clouds. FM 3-101-6 was written without the advantage of knowledge gained from trained operators using the tool in a realistic battlefield situation. Information gained from the Bio-Sim study was expected to generate substantial and useful improvements to the document, thus providing increased protection of U.S. troops against the threat of biological weapons.

\footnotetext{
${ }^{1}$ Editor's note: The quality of this image is not good, being taken from a low-resolution (NTSC) standard videotape. Unfortunately, this was the only image cleared for publication by the Soldier Biological Chemical Command so the author was not able to provide a better image.
} 
A second Bio-Sim study objective was to discover the characteristics of situations that most limited the use of the LR-BSDS. This objective arose from the need to understand situational characteristics or inherent shortcomings of the LR-BSDS that might limit its effective use. These limiting factors were not discussed in FM 3-101-6, again due to lack of testing the tool with trained operators in realistic battlefield situations.

The third primary Bio-Sim study objective was to demonstrate that networked simulation in a DVRE is a viable method for experimentation and training of tools such as the LR-BSDS. This objective arose from the requirement to better understand the role of simulation in training and testing of military systems. Newer versions of the LR-BSDS will drive the need for training on those devices. Real-world training can be prohibitively expensive, so training in simulation could be warranted with successful results from the Bio-Sim study. Furthermore, refinements in new versions of the LR-BSDS could be proposed from results gained via missions run in simulation.

\subsection{BIO-SIM PHASE III}

Phase III of the Bio-Sim study consisted of a set of comprehensive bio-cloud detection missions flown by trained LR-BSDS operators and helicopter flight crews. The missions were flown in a DVRE, using training equipment at Fort Rucker Aviation Testbed. The total number of Phase III trials was limited to 35 , which were run over a period of four weeks. Five different variables were manipulated during these trials (See Table 1). In addition, three different "vignettes", or terrain areas, were used for the irregular terrain so that the military participants would be less able to "game" the missions.

\begin{tabular}{|l|l|}
\hline Variable & Values \\
\hline Terrain Type & Smooth, irregular \\
\hline Standoff Distance & $5 \mathrm{~km} @$ @ 200 ft, 15km @ 1500 ft, 20km @, 5000ft \\
\hline Visibility & $15 \mathrm{~km}, 23 \mathrm{~km}$ \\
\hline Release Type & Ground, airborne \\
\hline Cloud Age & Continuous from 0:00:00 to 0:30:00 \\
\hline
\end{tabular}

Table 1: Phase III Variables and Values

\subsection{PHASE III SIMULATIONS}

Phase III of the Bio-Sim study used several simulations and simulators (See Figure 2) to model the real world systems that soldiers use and face when flying real bio-cloud detection missions. The LRBSDS was modeled using the BDT (BSDS Distributed Trainer). The BDT uses computer hardware and software to train LR-BSDS operators. The helicopter pilots flew the Phase III missions using a UH-60 training simulator cockpit. The BDT was housed in this cockpit shell, although not in its required location in a real helicopter. The U.S. military's ModSAF DVRE was used to generate and display the participants and the terrain for each mission, and also to provide the enemy air defense threat. The biocloud was generated using the NCBR (Nuclear, Chemical, Biological, and Radiation) cloud simulator. The NCBR also modeled the dispersion of the cloud and its interaction with the terrain. Simulyzer software was used to log the events of each trial for later playback. The Aviation Testbed provided the simulated cockpit communications hardware that allowed the LR-BSDS operators to communicate with the helicopter crewmembers. A MaK, Inc. "stealth" entity was injected into the DVRE simulation and was used to give experiment observers a "flying carpet" viewpoint of the mission. Finally, a communications channel was simulated that allowed the LR-BSDS and helicopter crews to speak with the "battlemaster", who played the role of an intelligence officer in charge of tracking the bio-cloud. 


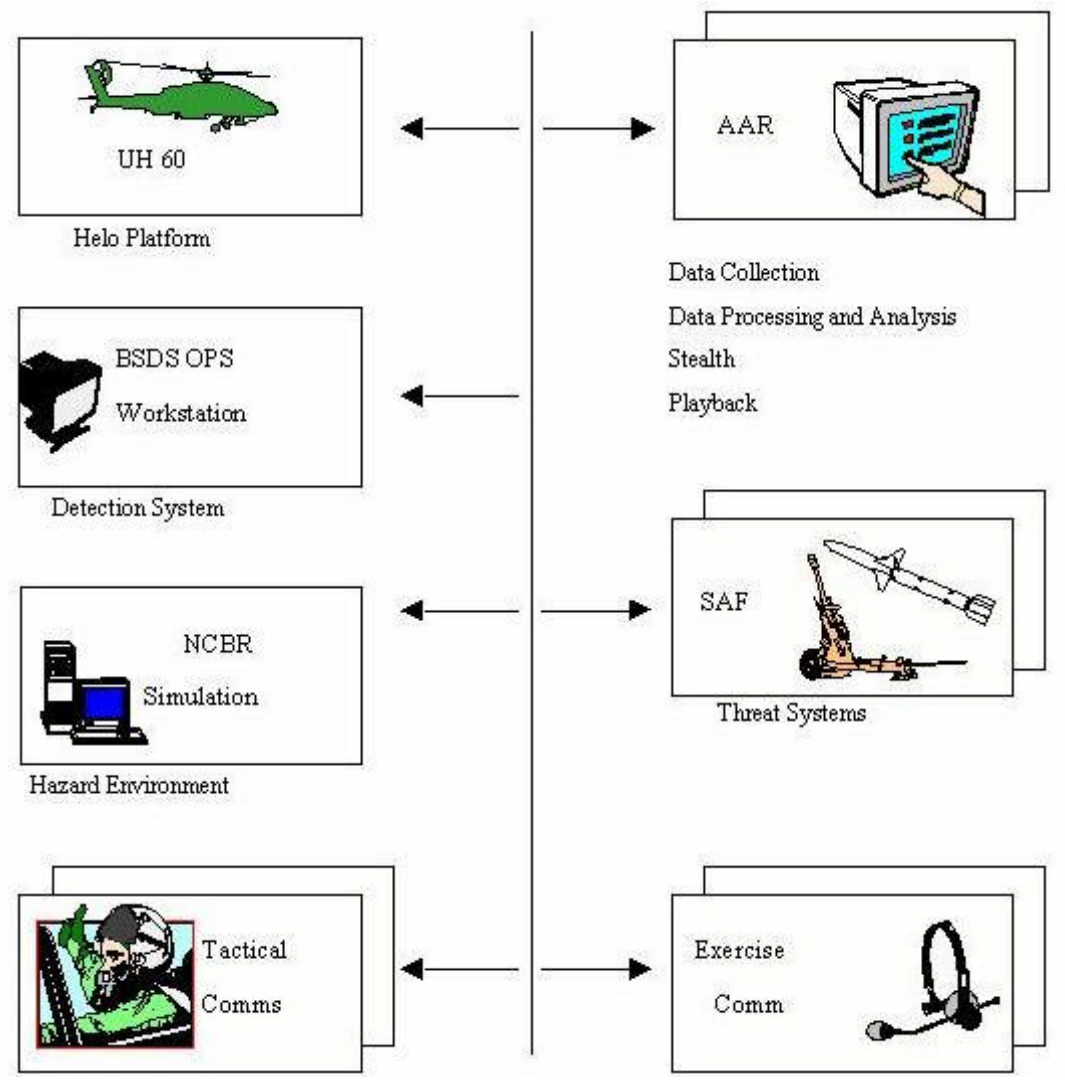

Figure 2: Phase III Models/Simulations. Left to right and down are as follows: UH 60: helicopter simulator, BSDS OPS Workstation: BDT (LR-BSDS training device), NCBR Simulation: bio-cloud dispersion simulator, Tactical Comms: intra-helicopter communications simulator, AAR (After Action Review): entity datalog recorder (Simulyzer), SAF (Semi-Automated Forces): enemy air defense simulator, Exercise Comms: "battlemaster" communication simulator

When these many simulations were combined to run a bio-cloud detection mission, the result in the DVRE was a helicopter flying above a virtual landscape. The LR-BSDS laser appeared as a line of light shining out of the side of the helicopter (although the LR-BSDS laser is not visible with the naked eye). The bio-cloud appeared as a smoothed and multi-faceted polygon moving over the terrain surface. When the LR-BSDS laser pierced the bio-cloud and the bio-cloud concentration was above the calculated detectability limit, the BDT screen would display a signal corresponding to the same signal as would appear if a real LR-BSDS had pierced a real bio-cloud. The trained LR-BSDS operators would then, assuming they saw and recognized the signal, call in a "bio-cloud detection" over their communications channel. This bio-cloud detection was received by the battlemaster in charge of the mission, similarly to the manner in which an intelligence operator situated far back from the front line of the battlefield would receive a real bio-cloud detection. 


\subsection{PHASE III DATA COLLECTION}

Data was collected in three formats, digital, video, and paper, due to the anticipated needs of the data analysis process.

Digital data took the form of a datalog file of the position signals that were generated by every entity during every trial, a spreadsheet of time-stamped key events (mission start time, bio-cloud release, first bio-cloud detection, etc), and a ModSAF screen capture (see Figure 3). The datalog was stored in case later playback of the mission was required. The key events were used extensively during the quantitative analysis, to calculate the elapsed times associated with particular key mission events. The ModSAF screen capture was used during the data analysis to confirm the starting physical location of various trial entities, and during presentations to indicate the general physical layout of the entities of each trial.

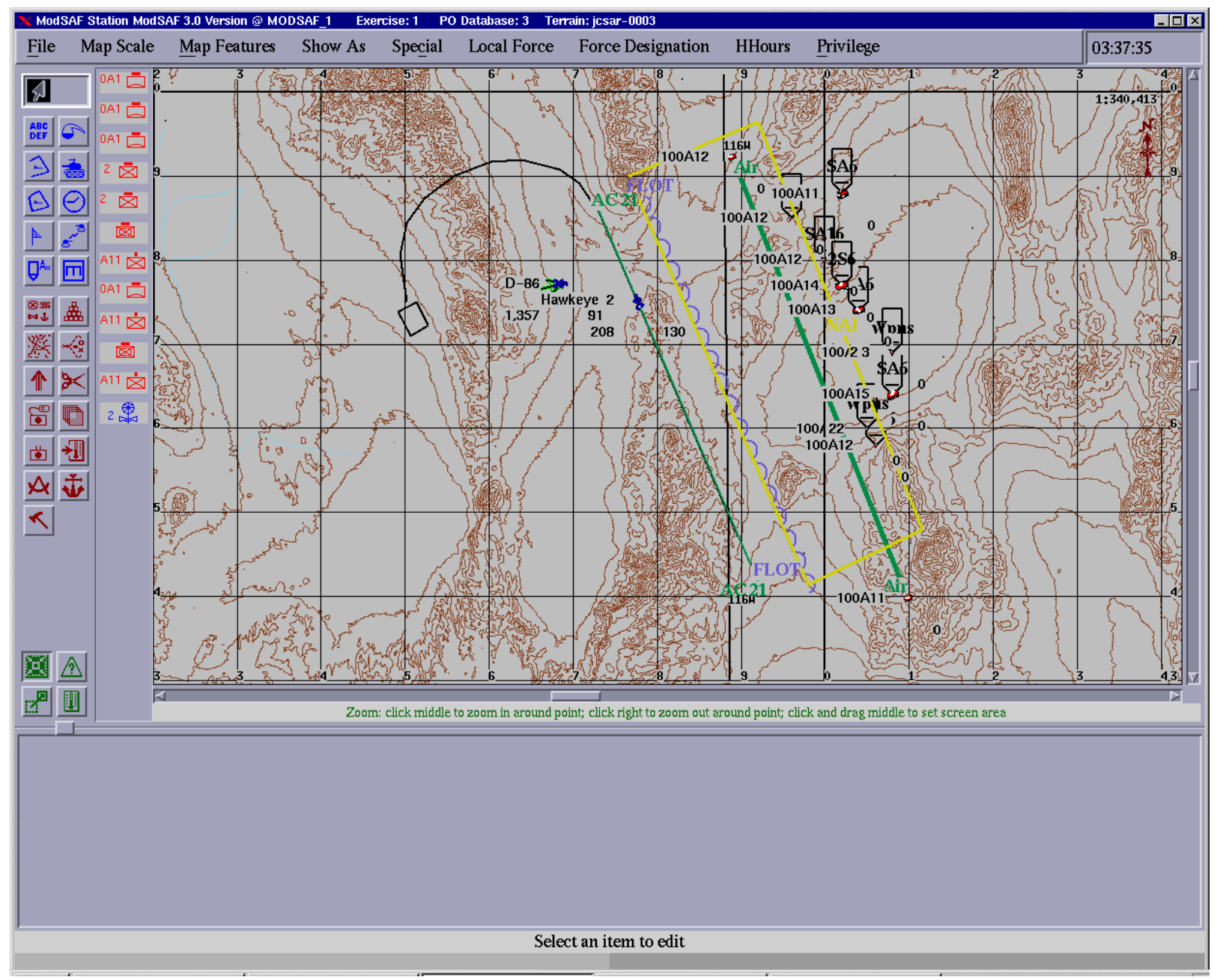

Figure 3: ModSAF Screen Capture 
Three different videotape recordings were made during each trial (starting with trial \#7, prior to which the NCBR screen was not captured). These video captures were: 1) in-the-face of the LR-BSDS operators, 2) the LR-BSDS screen (see Figure 2), and 3) the NCBR screen. These videotapes were used largely for documentation purposes, although all three were used at various points during the data analysis to more precisely determine the actions and timing of particular mission events.

Paper documents contained the comments and notes of all of the Phase III mission participants and observers. Paper-based notes were recorded for each mission's: 1) LR-BSDS and helicopter crew questionnaires, 2) cockpit observer, and 3) test director. At the end of each mission, both the LR-BSDS and helicopter crew filled out questionnaires about both their own demographic information and about their general feelings of that particular mission. During each mission, an "invisible" cockpit observer sat inside the cockpit and watched the LR-BSDS and helicopter crews, and noted their actions and communication processes. The last paper document of each mission was the set of comments made during each mission by the test director, who sat in a "stealth room" near the helicopter cockpit simulator. From this stealth room, the test director (and others in charge of supporting the Phase III simulations) could observe the actions of the mission participants as well as the actions taken by those supporting the simulations.

\section{Limitations}

Experimental assumptions and limitations must be enumerated before empirical results can be presented. This is important in DVRE simulation experiments because assumptions and limitations can have a large bearing on the extent to which the results may be extrapolated or applied to the real world. In the case of the Bio-Sim Phase III trials, there were a few assumptions/limitations.

\subsection{DETECTION LIMITS}

There have been no conclusive field studies that link the detection limits of the real world with the signal produced by the BDT when used in simulation. This restriction prevents the results of the study from indicating precise detection points and signals. That is, the study results indicate points at which the LR-BSDS operators detected a bio-cloud signal, but those signals, and therefore the detection indicated by the LR-BSDS operator cannot be confirmed as being identical to the signals that would have occurred in the real world on an actual LR-BSDS. This "correlation to the real world" is a standard problem in DVRE simulations.

\subsection{CLOUD DISPERSION}

The NCBR simulator modeled the dispersion of the bio-cloud and how it would flow around terrain features. However, the enormous complexity, randomness, and chaos that occur in the real world cannot be fully modeled by any current simulator. Therefore, the effects of meteorology and terrain in the simulated world are much more simple than they would be on a real cloud in the real world. This problem arises when attempting to model the movement of any entity made up of millions of parts/particles, such as water, sand, trees, clouds, and so forth. 


\subsection{LIMITED STATISTICAL VALIDITY}

Expenses limited the number of trials to 35 at the Aviation Testbed. This is far too small of a sample size for the number of parameters (five) and parameter value combinations (48, even considering that cloud age might only take on values of "young" or "old"). A full-factorial study would require that a minimum of 48 trials be run just to have one sample for each of the different combinatoric parameter value possibilities, and even more trials if the cloud age parameter had more than two discrete values. To derive statistically significant results related to each one of the different parameters would require even more trials. Therefore, the results of Phase III of the study must be considered with this statistical bound. This is also a problem with DVRE simulations that bring together many people and many expensive pieces of hardware and software.

\subsection{REAL-WORLD:SIMULATOR DIFFERENCES}

The physical relationship between the laser and flight crews in the real helicopter is such that they cannot directly see or communicate with each other. Due to the constraints of the helicopter cockpit simulator at the Aviation Testbed, the LR-BSDS crew was in close physical proximity to the helicopter pilot and co-pilot. Although they were instructed to ignore each other, it was possible for them to sense what the others were doing. Also, there are always significant differences between a military training simulator and the real piece of equipment that it replicates. The UH-60 training helicopter simulator was limited in its ability to display the environment outside of the helicopter, and did not have the same "touch and feel" of the flying characteristics of a real helicopter. Again, these problems arise in DVRE research when decisions must be made concerning the level of fidelity necessary to produce useful results.

\subsection{WEATHER EFFECTS}

ModSAF could not inject real-world weather phenomena into the Phase III trials. Although wind effects may have a large impact on the dispersion, and therefore detection of a bio-cloud, ModSAF cannot portray wind effects to the helicopter entity. The NCBR model did portray wind effects and their impact on bio-cloud dispersion and transport. This is also a standard problem in DVRE research when an attempt is made to model weather phenomena.

\section{Results}

The analytical results of the Bio-Sim study fell into three primary categories: 1) situational characteristics that led to improved use of the LR-BSDS, 2) situational characteristics that limited the effectiveness of the LR-BSDS, and 3) limitations of DVRE simulation for experimentation purposes. This section will cover only those results related to the third category.

Based on the comments derived from the Bio-Sim Phase III human-in-the-loop bio-cloud detection trials, several issues arose that had a effects on running the experimental trials. These issues may have had an impact on the results obtained from the trial missions. 


\subsection{IMPOSSIBLE ACTIONS}

On occasion, LR-BSDS crew or helicopter flight crewmembers traded places. During a real mission in a UH-60, neither the LR-BSDS nor flight crewmembers will be able to change their physical location within the airframe. The LR-BSDS crew would sometimes look out the "side windows" of the helicopter simulator to use visual cues of the terrain to scan more efficiently. This will rarely if ever be possible during a real mission, and impossible during night missions. Toward the end of the Phase III trials, the pilots would occasionally look at the LR-BSDS screen, while the laser crew would sometimes look at the pilots "moving map" display. Both of these actions are impossible in the real helicopter configuration. Finally, some communication occurred between the laser and flight crews without use of the radio communication system. This is also impossible in a real UH-60.

\subsection{WEATHER EFFECTS}

Although wind effects have a large impact on the dispersion, and therefore detection of a bio-cloud, ModSAF cannot portray wind effects to the helicopter entity. The NCBR model did portray wind effects and their impact on the bio-cloud.

\subsection{REAL-WORLD:SIMULATOR DIFFERENCES}

The pilots sometimes complained about the difference between the control characteristics of the simulator and real helicopter. In particular, they mentioned the difficulty of getting realistic visual cues from the large-screen video monitors that simulated the view out of the cockpit. Maneuverability of the helicopter simulator was also described as much more sluggish than in the real world. Finally, the method of keeping mission time (by gallons of fuel burned/left) in the helicopter simulator was appropriate, but apparently numerically far off of what would occur in a real helicopter.

\subsection{LR-BSDS DISPLAYDIFFERENCES}

The first of these display differences appeared as an upward curve of the actually flat terrain in the lower LR-BSDS window pane when the LR-BSDS operator used positive roll to scan out further from the helicopter. It is unknown if this is also the case in the real device or if it is an artifact of using the simulator. The second of these differences indicated that a faint cloud, when displayed on the LR-BSDS simulator screen, appeared to look differently than how it would on the screen of the actual device. The third of these differences happened when the laser crew was scanning out over a long distance and using roll correct at the same time. This caused the ground return in the LR-BSDS lower window to appear to be sloping downward away from the helicopter, although this was not actually the case. The last difference occurred very frequently and was exemplified by a significant difference between the terrain displayed in the laser up-scan and down-scan in the LR-BSDS waveform window. In many instances, the terrain shown in the waveform window during the laser up-scan looked very different than the terrain shown during the down-scan. The terrain that was displayed was obviously the same piece of ground, yet as represented by an image that did not overlap much at all from one up-scan to the next down-scan. 


\subsection{AIR DEFENSE THREATS}

Those Phase III mission participants who had battle experience (mostly flight crew members) indicated that the air defense threats in the simulated environment was far more extensive in range and overwhelming in power than they would be in a real battle.

\subsection{SPURIOUS EVENTS}

On two occasions, missions were affected by events that were of unknown origin. The first of these caused momentary loss of control of the helicopter; the second caused a problem with the view out of the helicopter.

\section{Conclusions}

The general conclusions derived from the Bio-Sim Study fall into three categories. The first concerns the value of performing human-in-the-loop trials in a DVRE simulation. The second relates to updating the FM 3-101-6 document based on lessons learned during the Bio-Sim study. The third pertains to training and communications issues. This paper will consider only those conclusions relating to the first (DVRE simulation) category.

\subsection{HUMANS-IN-THE-LOOP}

Human-in-the-loop trials are a useful way to generate insightful details about a process. Some knowledge can be derived using purely computer-based constructive experiments. However, the use of real humans in an investigation of any system in which humans play a significant role will provide information that cannot be discovered using only computer-based processes. The Bio-Sim Phase III DVRE simulation trials did exactly that.

\subsection{SIMULATION}

Using real operators and state-of-the-art simulations during the Phase III trials allowed data to be gathered which more precisely defined those battlefield characteristics that enhanced or limited use of the LR-BSDS. Also, the Phase III trials showed that simulation could be used for such experimental purposes as well as for detailed training. While the use of simulation places limits on extrapolating the experimental results, much was learned about where simulations could be improved for better future experimentation. Changes to the general LR-BSDS training process were proposed, and support for the use of simulation for training was justified.

\section{Recommendations}

This section specifies the recommendations that were proposed [Brooks and Beckman, 2000] as a result of the outcomes of the Bio-Sim study. The recommendations fell into five main areas: 1) training and training-related issues, 2) communication that occurs within the helicopter, 3) tracking the bio-cloud location and movement within the helicopter, 4) tasks associated with cloud scanning, identification, and tracking, and 5) use of DVRE simulation in an experiment such as the Bio-Sim study. Again, this paper covers only those recommendations relating to the fifth area (DVRE simulation). 
Recognition must be given to the differences between the simulated and real environments and to the extent that the results have been impacted by those differences. However, future experiments must consider the discrepancies between the real world and the models used to simulate it. Although there will always be differences between the real world and models, those differences may be acceptable. Unfortunately, there is almost no academic or practical research that suggests which models will have the most influence on experimental results. This does not, however, remove the necessity of recognizing that such differences do exist. The Bio-Sim Phase III trials did show that applying a multitude of models to perform experiments and training using a complicated military human-system interface could provide acceptable and useful results.

\section{Summary}

The following section describes the high-level summary statements derived from the results of the Phase III trials, and that are related to three areas. These areas are: 1) use of humans in testing, 2) impact of simulation on testing, and 3) areas of future research. Justification for the user of humans in the testing process comes from years of research in the arena of consumer product testing. Consumer product researchers have found that the best way to improve consumer products or systems is to watch users operate them in situations that are as realistic as possible. Recognition of the impact of using DVRE simulation for military testing came from watching the LR-BSDS and helicopter crews performing biocloud detection missions. At various points during these missions, instances were detected during which the imperfect Phase III simulations (all simulations are imperfect) impacted crew operations. Suggestions for future research directions came from watching the LR-BSDS and helicopter crews sometimes unsuccessfully deal with the issue of deciding what information was necessary to maintain or control within the helicopter. No conclusions were reached related to this topic area, as the Bio-Sim study was not constructed to investigate uses of information at higher levels of battlefield command and control.

\subsection{HUMANS-IN-THE-LOOP}

It would be possible to use purely computer-controlled models to do testing and run experiments investigating use of the LR-BSDS. However, there is an extraordinary amount of additional information to be learned by using actual trained LR-BSDS and helicopter crews. They can be put in situations that force them to use their training and talents in novel ways that constructive models cannot. Their performance will most accurately replicate the actions of real soldiers, as they perform better and worse in a variety of battlefield circumstances.

The Phase III trials indicated that crew performance was better in those battlefield conditions in which they had practiced communicating with each other. This is a significant point, as currently, the LR-BSDS and helicopter crew do not train together. Furthermore, there is not currently a dedicated set of helicopter pilots trained specifically for the bio-cloud detection mission. The crew also performed better when both crews had previous experience with "unusual" situations. These types of unusual conditions arise when the bio-cloud is released at an angle to the front battle line, where some extreme meteorological condition exists, where some type of terrain feature precludes direct detection of the biocloud, or where a combination of these factors exists. Without prior exposure to these types of confounding influences, cloud detection was much more difficult.

The crew performed worse when first confronted with the unusual conditions mentioned above, when the action was very fast-paced, or when the mission grew tedious. The first case arose regularly, as terrain features often confronted the crew, making their detection task more difficult. However, as they became accustomed to dealing with these terrain features, their performance increased. When action 
within the helicopter was frenetic, often due to numerous actions requiring immediate attention, performance decreased. This was a direct result of the physical and cognitive overload that occasionally arose during a mission. This is a relevant point, as the DVRE simulation was able to provide a somewhat realistic environment. However, no simulation can yet generate environmental cues as rich as those that occur in the real world. Therefore, the physical and cognitive overload that occurred during the Phase III trials is likely to be even less than what would occur during a real bio-cloud detection mission.

Finally, crew performance suffered when the mission became boring. Unfortunately, this occurred during many missions, and is likely to be a common occurrence during real-world missions. There are many times during the bio-cloud detection mission (sometimes the entire mission) where essentially nothing happens. It is during these times that the LR-BSDS crew would lose focus on their assigned tasks and miss bio-cloud detections.

\subsection{DVRE SIMULATION ISSUES}

This is an important subject because simulation allows the experimenter and trainer to create situations more focused and more often than would arise in the real world. The downside is that simulations are all abstractions of some real world system, and therefore do not replicate those systems in complete detail. The extent to which a simulation models the real-world system details will determine the extent to which the experiment (or training) is applicable to the real world.

Each of the Phase III simulations modeled their corresponding real-world system to a different extent. These differences are partly due to the financial extent to which the builder of each simulator went in order to replicate the real world. However, in the case of some simulators, the differences are also partly due to the lack of knowledge of the real world. Specifically, there are gaps in the knowledge of exactly how a cloud particle is created, disperses, and is concentrated in the real world. There are also gaps in the knowledge of how meteorological effects impact the bio-cloud and how the LR-BSDS laser interacts with a cloud particle to produce a return signal. These variances impact training through such events as screen displays that are not identical to those that would be seen in the real world. They also impact experimentation by limiting the extent to which experimental results can be extrapolated to the real world. For example, signals that appear too strongly in the simulated world produce cloud detections that might not occur in the real world. There are also differences in the simulated physical workspace environments of the LR-BSDS and helicopter crews. These differences operate in both performanceenhancing and performance-limiting ways. They improve mission performance through such impacts as allowing the helicopter pilots to see the world without the effects of weather or night. They reduce performance in such ways as diminish the helicopter pilot's view of the outside world due to the limited resolution monitors that act as their cockpit view.

\subsection{FUTURE RESEARCH DIRECTIONS}

The primary goal of the Bio-Sim study was to investigate use of the LR-BSDS as a tool, and to use that knowledge to improve the FM 3-101-6 document. However, during the Bio-Sim study, and particularly during the Phase III trials, it became obvious that information (particularly about the location of the bio-cloud and who had that information) might play a more important role than previously estimated. This section deals with the understanding and use of the role of information and information processing in the bio-cloud detection mission, and how future experimentation might increase that understanding.

In particular, an area of information control that was not examined during the Bio-Sim study was knowledge of the position and movement of the bio-cloud and who should have that knowledge. In early Phase III missions, the helicopter and LR-BSDS crews made no attempt to track the location of the bio- 
cloud. They presumed that their entire mission was to detect and track the cloud, and pass that information on to higher levels of control. As the participants became more familiar with the bio-cloud detection mission, however, they also became aware of points in a mission where they might be exposed directly to the bio-cloud by flying into it. At this point, they began to make rudimentary attempts to track the bio-cloud position and movement within the helicopter. It is not outside the realm of their responsibilities to do this; however, they were not given this task as a requirement, nor were they given tools to make cloud-tracking easier.

A second major area of information processing and control that was not investigated during the Bio-Sim study concerned information passed on to higher echelon levels. The Phase III trials assumed that there was no higher-level chain of command (other than the battlemaster) to record and process biocloud information. Therefore, no investigation was made into the amounts and/or formats of information that might be most useful to those higher levels of command. There is a growing field of research that examines more useful formats of information that can be provided to users based on those users individual and role traits, and on the characteristics of the tasks they are attempting to complete.

Future research might be more valuable when focused on 1) the utility of tracking the location and movement of the bio-cloud by participants within the helicopter, 2) the amounts and formats of bio-cloud information that is passed on to higher levels of command and control, and 3) the impact on LR-BSDS and flight crew actions when someone in the helicopter tracked the location and movement of the biocloud.

\section{Acknowledgments}

This research was support in full by funds from the Bio-Sim study sponsor, the United States Army's Soldier and Biological Chemical Command.

\section{REFERENCES}

Mendonsa, R.A.: "LIDAR Protects Against Biological Warfare Agents", Photonics Spectra, February, 1997.

Condatore, L.A., Guthrie, R.B., Bradshaw, B.J., Logan, K.E., Lingvay, L.S., Smith, T.H., Kaffenberger, T.S., Jezek, B.W., Cannaliato, V.J., Ginley, W.J., and Hungate, B.S.: "U.S. Army Soldier and Biological Chemical Command counterproliferation long-range biological standoff detection system (CP LRBSDS)", Proceedings of SPIE--The International Society for Optical Engineering, Vol. 3707, p. 188-196, May, 1999.

Brooks, P., and Beckman, P.: "Bio.Sim Study Findings and Analysis", Proceedings of the 68th MORS (Military Operations Research Society) Symposium, June, 2000. 


\section{AUTHOR BIOGRAPHY}

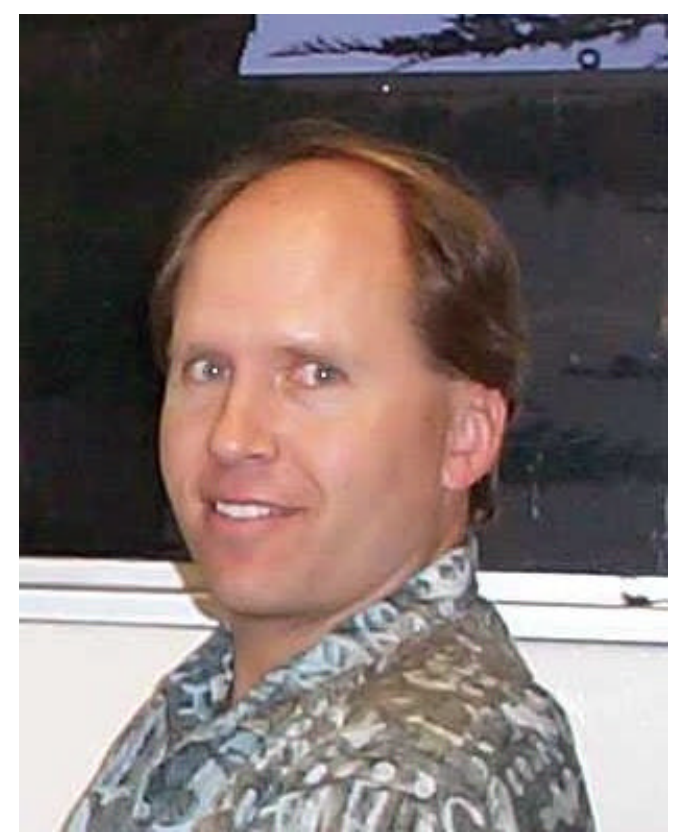

Paul Beckman is an Assistant Professor in the Information Systems and Business Analysis Department in the College of Business at San Francisco State University, and an adjunct research staff member at the Institute for Defense Analyses. His research interests are in the areas of high-fidelity user interfaces, virtual environments, human performance measurement, and military training systems.

\section{Contact information:}

Email link: mailto:pbeckman@sfsu.edu 